Advances in Complex Systems

(C) World Scientific Publishing Company

\title{
AVOIDING EXTINCTION BY MIGRATION: THE CASE OF THE HEAD LOUSE
}

\author{
OCTAVIO CABRERA \\ DAMIÁN H. ZANETTE * \\ Centro Atómico Bariloche and Instituto Balseiro \\ 8400 San Carlos de Bariloche, Río Negro, Argentina \\ cabreraoct@gmail.com,zanette@cab.cnea.gov.ar \\ Received (received date) \\ Revised (revised date)
}

\begin{abstract}
The possibility of spreading by migration, colonizing new spatial domains suitable for development and reproduction, can substantially relieve a biological population from the risk of extinction. By means of a realistic computational model based on empirical data, we study this phenomenon for the human head louse, Pediculus humanus capitis. In particular, we show that a lice colony infesting a single isolated host is prone to extinction by stochastic population fluctuations within an interval of several months, while migration over a relatively small group of hosts in contact with each other is enough to insure the prevalence of the infestation for indefinitely long periods. We characterize the interplay of the size of the host group with the host-to-host contagion probability, which controls a transition between extinction of the lice population and a situation where the infestation is endemic.
\end{abstract}

Keywords: Population dynamics; migration; Pediculus humanus capitis.

\section{Introduction}

The evolution of the number of individuals in a large, spatially homogeneous biological population can be well approximated by smooth functions of time, governed by a system of ordinary differential equations. The trend of the population to flourish or decline results from the balance of average birth, death, and migration rates which, ordinarily, are in turn smoothly time-varying quantities [1]. Spatial inhomogeneity can readily be incorporated to this kind of continuous description by means of partial differential equations [2].

On the other hand, small populations are likely to undergo sharp variations in size even over short time spans, due to the effect of random fluctuations 3 . For instance, a rapid succession of deaths of fertile individuals may lead to a bottleneck that severely impairs growth of the population in the short term, and perhaps

*Also at Consejo Nacional de Investigaciones Científicas y Técnicas, Argentina. 
induce its extinction. The smaller the population, moreover, the more pronounced the effect is: under homogenous environmental conditions and taking into account stochastic death events only, the probability that all the members of a population of $N$ individuals die within a given period is $p_{N}=p_{1}^{N}$, where $p_{1}$ is the death probability of a single individual during the same time. As a consequence, for example, if a group of animals becomes extinct with a probability of 0.0001 during the non-breeding season, a similar group of half its size becomes extinct with a probability of 0.01 . The extinction of the second group is, therefore, a hundred times more likely.

A significant illustration of the risk of extinction that threatens small populations is given by the fact that the main criterion to define endangered animal and plant species is, precisely, their reduced number of individuals [4. Another major criterion of extinction risk regards the limited extension and high fragmentation of their geographical distribution. In contrast, under favorable conditions, the capability of expanding the distribution by colonizing new regions may guarantee access to the resources necessary for the population to grow in size, thus decreasing the exposure to extinction. Even a geographically fragmented population can avoid local extinction by timely migration between different habitat patches [5]. If, nevertheless, extinction in a patch does occur, it can be later recolonized by individuals of the same species coming from other zones.

In this paper, we address the possibility of avoiding population extinction by colonization of new spatial domains in the specific case of the human head louse, Pediculus humanus capitis. Recent gathering of empirical results on the louse's life parameters made it possible to put forward realistic mathematical models for the demography of lice colonies [6], on which we base our analysis. In a single host's head, the number of lice at any given moment is limited to, at most, a few tens of individuals. The host's self-grooming, which becomes more intense as the number of lice grows, controls the proliferation of nymphs and adults. Moreover, the number of eggs laid by each fertilized female during its whole lifespan is relatively small. As a result, as we show here, the average waiting time for a single-host lice colony to become extinct by random fluctuations - disregarding any kind of specific treatment - is typically within the order of a few to several months. The possibility of migrating from one host to another, thus establishing new colonies, is therefore crucial for the survival of the infestation over much longer time spans.

After formulating our computational model on the basis of empirical observations of the louse's life cycle, we first present extensive numerical simulations for single-host colonies, characterizing their survival probability and average duration as functions of the parameters associated to the host's grooming. Then, we move to the case of several hosts, where we find our main result: for a fixed rate of migration between hosts, there is a minimum number of hosts above which the lice population survives indefinitely, the infestation thus becoming endemic. Conversely, for a group of hosts of a given size, the infestation becomes endemic if the migration rate is large enough. The transition between extinction and endemic prevalence is characterized numerically. Moreover, we show that it can be qualitatively explained by means of 
a simple metapopulation model. Conclusions and perspectives are drawn in the last section.

\section{The life of lice: Empirical data and computational model}

Lice are parasitic insects, belonging to the genus Pediculus among others, that infest almost every bird and mammal species. Pediculosis, the infestation of lice, has been a steadfast companion of mankind until powerful insecticides began to be used against it by the mid twentieth century. The ensuing decrease in the prevalence of pediculosis led head-louse infestation, its most common variety, to be regarded as a signature of lack of hygiene and precarious life conditions. In the developed world, pediculosis thus became a kind of social taboo, which also resulted into a relative loss of interest from health scientists. Systematic studies of the biology and epidemiology of lice had to wait a few decades, until pediculosis regained prevalence - probably due to the combined effect of lice's developed resistance to insecticides and the banishment of the most aggressive chemicals from treatment [7].

In the last decade, a few controlled studies have quantitatively characterized the life cycle of the louse $[8,9$. These results have recently been used to formulate a mathematical model for the population dynamics of a lice colony [6]. The data relevant to our contribution can be summarized as in the following.

\subsection{Life history of the head louse}

As for many other insects, the life of a louse spans three stages: egg, nymph, and adult. The nymph, in turn, undergoes three moults before becoming an adult. Observations of in vivo and in vitro lice colonies show that some $76 \pm 3 \%$ of the eggs are viable and bear a nymph, each of them hatching $8.4 \pm 0.1$ days after it was laid. Recorded durations of the egg stage vary between 7 and 11 days. The duration of the nymph stage is even better defined, with recorded values of 8 and 9 days, depending on sex and strain [8.

In cultivated colonies, some $23 \%$ of the nymphs die before reaching adulthood. The adult stage, in turn, lasts 12 days on the average, with a maximal recorded duration of 23 days [8]. Under natural life on a host, mortality rates grow due to the host's self-grooming, mainly as combing, washing and scratching. Grooming, on the other hand, does not affect eggs, which are firmly glued to the hair. Both nymphs and adults are mobile and feed on their host's blood several times a day [9. Consequently, they survive only a few hours if they are taken away from the host.

After the first or second day of adulthood, each fertile female lays $4.9 \pm 0.2$ eggs per day [8]. Scattered studies starting at the nineteenth century suggest a femalebiased sex ratio of around $60 \%$ [10. Plausibly, a single adult male suffices to fertilize all the adult females in a head's colony which, under normal conditions, consists of 10 to 20 mobile (nymph plus adult) individuals. It has been moreover suggested that, once fertilized, a female remains fertile for its whole life. 
Pediculosis mostly affects children, from the age of 3 to the beginning of puberty. Hundreds of millions of children worldwide are affected each year. Females seem to be more prone to get infested. In any given social group of children - for instance, at school or in sport activities - the typical prevalence ranges between 2 and $20 \%$ 7]. It is apparent from direct observation of such groups that contagion plays a crucial role in the dynamics of head lice infestations. Transmission from head to head occurs by direct motion of the lice when the hosts are close enough to each other, or by transportation on inanimate objects such as combs, clothes, and toys 11, 12. Symptoms of pediculosis - mainly, itching caused by allergic reaction to the lice bites - appear when the number of mobile individuals reaches a certain threshold, which has been estimated to be around 15 lice [6]. This triggers more intense grooming and, consequently, nymph and adult mortality increases.

Quantitative results on the transmission dynamics of head lice in realistic conditions - making it possible, for instance, to assign numerical values to host-tohost contagion probabilities- seem not to be not available yet [13, 14, 15]. It is conceivable, moreover, that such parameters are strongly dependent on specific circumstances of the host group, ranging from socioeconomic conditions and cultural traits to the kind of social activity they carry on together. The effectiveness of grooming below and above the awareness threshold, in turn, has not been measured. Similarly, ovicidal treatments - both chemical and with lice combs - are difficult to evaluate during actual use [16. This kind of treatment, in any case, is not considered in the present contribution.

\subsection{Computational agent-based model}

On the basis of the data presented above, we formulate a computational model for an evolving population of lice, according to the rules described below. Time elapses in discrete steps, each step corresponding to a day. Each louse is represented by an agent endowed with several attributes. An "internal clock," updated at every time step, measures the louse's age in days, starting at the day where the egg was laid by the mother. At any time, the louse's life stage (egg, nymph, or adult) is determined from its age, using the parameters reported below. A binary index defines the louse's sex (female or male). Another index records whether an adult female is fertilized or not. Finally, in populations distributed over several hosts, an additional index identifies the louse's present host.

In our model, as explained below, only viable eggs — namely, eggs which successfully bring forth a nymph - are taken into account. Hatching takes place for all eggs at day 9. The nymph stage, in turn, lasts 8 days, thus ending at day 17 . During this stage, there is a constant spontaneous death probability of 0.03 per day. Under this sole effect, on the average, the nymph population reduces to a fraction of approximately 0.78 during the whole stage. As explained below, however, this mortality must be increased with the contribution from death events caused by the host's grooming. 
During the adult stage, the probability of spontaneous death of a louse grows to 0.083 per day. If lice would be allowed to reach arbitrarily old ages, this would determine an average duration of 12 days for adults. For computational reasons, however, we limit the duration of this stage to a maximum of 23 days, so that all surviving lice die exactly 40 days after the corresponding eggs were laid.

Fertilization of a female requires that, at any day of its adult life, it shares its host with an adult male. Once this happens, the female remains fertilized for the remaining of its life. Fertilized females can lay eggs after the second day as adults. Every day, each one of these females lays 5 eggs. The viability probability of each egg is fixed at 0.76 so that, on the average, $3.8 \pm 2.1$ viable eggs per fertilized female per day are incorporated to the population. Nonviable eggs are discarded. The sex is determined probabilistically at the moment in which each egg is laid, with a female-biased sex ratio of $60 \%$.

We model the effect of the host's grooming as an addition to the death rate of nymphs and adults, assuming that ordinary grooming takes place below a certain awareness threshold, whereas above the threshold - when symptoms of the infestation appear - more intense grooming is triggered. When the total number of mobile lice in the host's head is less than a critical value $n_{c}$, their spontaneous death probability per day is increased by 0.05 , so that it reaches 0.08 for nymphs and 0.133 for adults. When, on the other hand, the number of mobile lice grows beyond the threshold $n_{c}$, death probabilities are added a further contribution $p_{c}$. Both $n_{c}$ and $p_{c}$ remain as free parameters, as we aim at studying how results on single-host infestation change when they are varied.

Finally, for populations distributed over several hosts, it is necessary to fix the per-day probability $p_{s}$ that a mobile louse passes from one host to another. This "jump" probability is also kept as a free parameter.

In all cases, the initial condition for the population consists of a single fertilized female in the third day of adulthood, inhabiting one of the hosts. At every time step in the computational realization of the model, the population is subjected to the above rules, according to their respective conditions, in the following order: (1) unfertilized adult females become fertilized, (2) newly laid eggs are added to the population, (3) the "internal clock" of all agents is updated, (4) agents are allowed to move from one host to another, and (5) death events are applied and dead agents removed.

By comparison with the mathematical model on which the present computational scheme is based [6], we are here being parsimonious with respect to the number of parameters, in particular, regarding the durations of the egg and nymph stages. Indeed, instead of admitting that the durations are statistically distributed - which requires to define the respective probability distributions - we assume that they are deterministically defined. This simplification, however, seems to be reasonably justified by empirical observations of the narrow variation of those durations (see Section 2.11). In our model, thus, the dispersion in the life span of individual lice and the increasing overlapping of successive generations is respectively controlled 
by mortality during the nymph and adult stages, and by randomness in the times of egg laying.

\section{Single-host colonies}

In this section, we study the population dynamics of a lice colony infesting a single host. The results set the basis for the analysis of populations on several hosts, when contagion is allowed for.

We first consider a lice population where the effect of the awareness threshold is disregarded. Death rates, hence, are those determined by the probability of spontaneous death of nymphs and adults plus the contribution of sub-threshold grooming. Although there is a small probability that the initial female dies without having laid any viable egg, thus terminating the colony during the first few days, the typical situation is that a growing population does develop. Since, in the absence of the awareness threshold, the number of birth and death events is on the average proportional to the number of lice in different life stages, it is expected that the population grows exponentially in time.

The upper panel of Fig. 1 1 illustrates this situation in a single realization of our computational model. Different symbols represent the recorded number of lice in each life stage as a function of time. In this log-linear plot, straight lines with positive slope correspond to exponential growth. We find that the three sub-populations asymptotically approach this kind of growth through damped oscillations. The period of these oscillations is approximately given by the age at which a fertilized adult female is first able to lay eggs, namely, 19 days after its own egg was laid. Oscillation damping is due to the effect of fluctuations in the duration of successive generations along each lineage, as the population progresses. Note that, asymptotically, the numbers of eggs, nymphs and adults represent fixed fractions of the total population, respectively, around $67 \%, 28 \%$, and $5 \%$. As indicated by the straight line in the figure, the average growth slope of the population equals 1.09 day $^{-1}$. In other words, the population increases by $9 \%$ every day. This is in reasonable quantitative agreement with other mathematical models for lice populations [6] which, under similar conditions, predict a growth rate of 12 to $13 \%$ per day.

Naturally, no human host is expected to endure in their head a lice colony that attains hundreds or thousands of individuals. The above results show, however, that - for the chosen set of parameter values - the trend of the lice population below the awareness threshold is to grow with time. The effect of introducing the threshold $n_{c}$, with an additional, sufficiently large death probability $p_{c}$, is precisely to inhibit the unlimited growth of the population. The lower panel of Fig. 1 shows an example for a single realization with $n_{c}=10$ and $p_{c}=0.3$ day $^{-1}$ (note the linear-linear scales). Initially, due to the succession of life stages, oscillations similar to those in the upper panel take place in the three sub-populations. Now, however, the number of mobile lice varies irregularly around the threshold. Since the sub-populations of nymphs and adults never attain very large sizes, fluctuations in the frequency of 

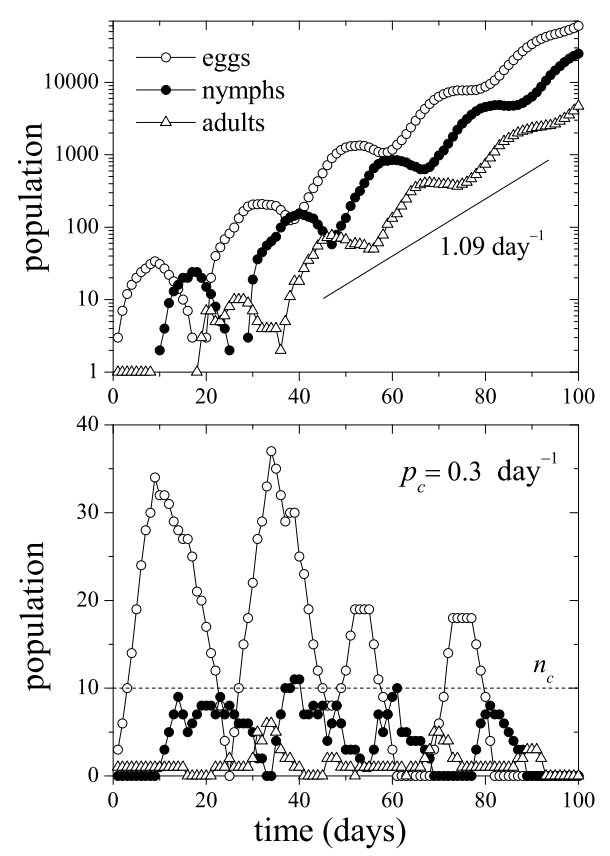

Fig. 1. Upper panel: Number of eggs, nymphs, and adults as a function of time (log-linear scales) in a single computational realization for a lice population where the host's awareness threshold is disregarded. The straight line stands for the average slope of the exponential growth. Lower panel: As in the upper panel (linear-linear scales), for a population with an awareness threshold of $n_{c}=10$ mobile lice, and an additional death probability due to grooming above threshold fixed at $p_{c}=0.3$ day $^{-1}$. The population becomes extinct by the death of the last adult at day 93 .

birth and death events eventually lead to the extinction of the whole colony.

In order to provide a statistical description of the time evolution of the population, we have performed series of $10^{5}$ realizations for each parameter set, averaging the number of lice over realizations at each time step up to a maximum of 3000 days ( $\approx 8$ years, the typical maximal duration of pediculosis during childhood). Figure 2 shows the average total population (eggs plus mobile individuals) as a function of time, for several choices of the parameters $n_{c}$ and $p_{c}$. We find that, after an initial stage with damped oscillations of a period of about 20 days - displayed in more detail in the insets - the average population settles down to an essentially monotonic time dependence, either increasing (for $n_{c}=20, p_{c}=0.15$; lower panel) or decreasing. This long-time behaviour is well approximated by an exponential (a straight line in the $\log$-linear scales of the plot).

The fact that, for fixed $n_{c}$ and depending on the value of $p_{c}$, the average total population grows or shrinks with time points to the existence of a critical value for $p_{c}$ above which the lice colony eventually becomes extinct. Analyzing in detail intermediate values of $p_{c}$, we have found that the critical probability is $p_{c}=0.17 \pm$ 


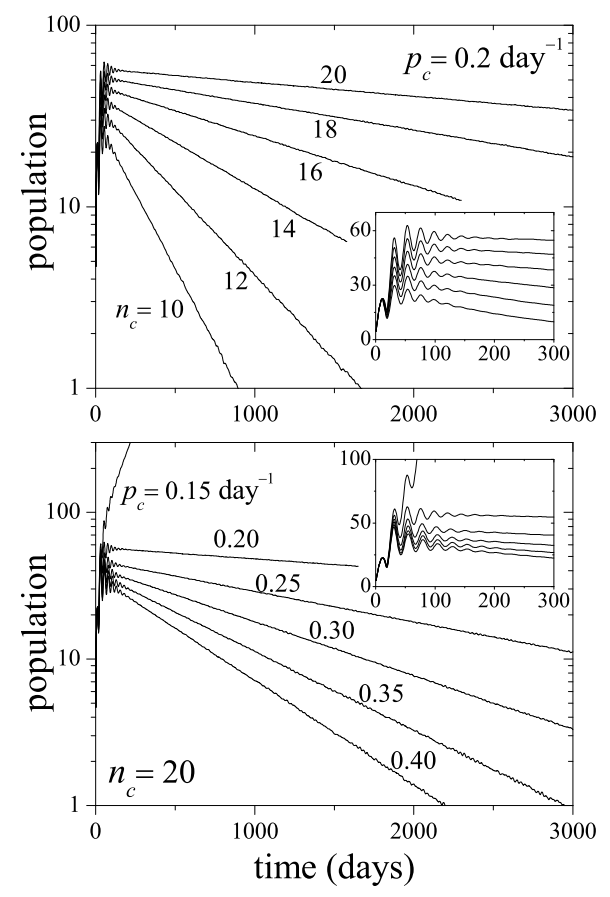

Fig. 2. Total population - eggs plus nymphs plus adults - as a function of time, averaged over $10^{5}$ realizations of the lice colony, for $p_{c}=0.2$ day $^{-1}$ and several values of the awareness threshold $n_{c}$ (upper panel), and for $n_{c}=20$ and several values of $p_{c}$ (lower panel). The insets show closeups for short times. Note that the main plots have log-linear scales, while in the insets they are linear-linear.

$0.01 \mathrm{day}^{-1}$ for awareness thresholds varying from $n_{c}=10$ to 20 . This critical value coincides with the estimation given in previous work [6]. As for its dependence on $n_{c}$, we expect that for unrealistically small and large values of the awareness threshold, $p_{c}$ becomes respectively lower and higher. In fact, for small $n_{c}$, even very small values of $p_{c}$ would lead to a rapid extinction of the colony due to fluctuations. On the other hand, large values of $n_{c}$ would permit the accumulation of many eggs even if the death probability of mobile lice above the threshold is very large.

The exponential decay of the average total population (for $p_{c}$ above the critical value) reveals that its long-time evolution would be well described by a linear equation, of the type $\dot{n}=-\alpha n$ with $\alpha>0$. As expected, our results show that the extinction rate $\alpha$ grows as $n_{c}$ decreases and $p_{c}$ increases. From the epidemiological viewpoint, however, it is important to provide a statistical quantification of the duration of the lice colony that includes both the long-time behavior and the initial stages of the evolution. To this end, we define the survival probability $P(t)$ as the probability that a population starting at $t=0$ is still extant at time $t$, i.e. that its 
whole duration is equal or larger than $t$. The survival probability can be written as

$$
P(t)=\sum_{u=t+1}^{\infty} p(u)
$$

where $p(u)$ is the probability that the population becomes extinct exactly at time $u$. Note that the average duration, $T=\sum_{u=0}^{\infty} u p(u)$, has a simple expression in terms of the survival probability, as

$$
T=\sum_{t=0}^{\infty} P(t)
$$

We have evaluated $P(t)$ from our series of $10^{5}$ realizations, for which we recorded the total duration of the population. The fraction of realizations where the duration is equal to $u$ gives a direct estimate of $p(u)$. Realizations where the lice population was still extant at the maximum time of 3000 days were counted and added to $P(t)$ for all $t \leq 3000$.
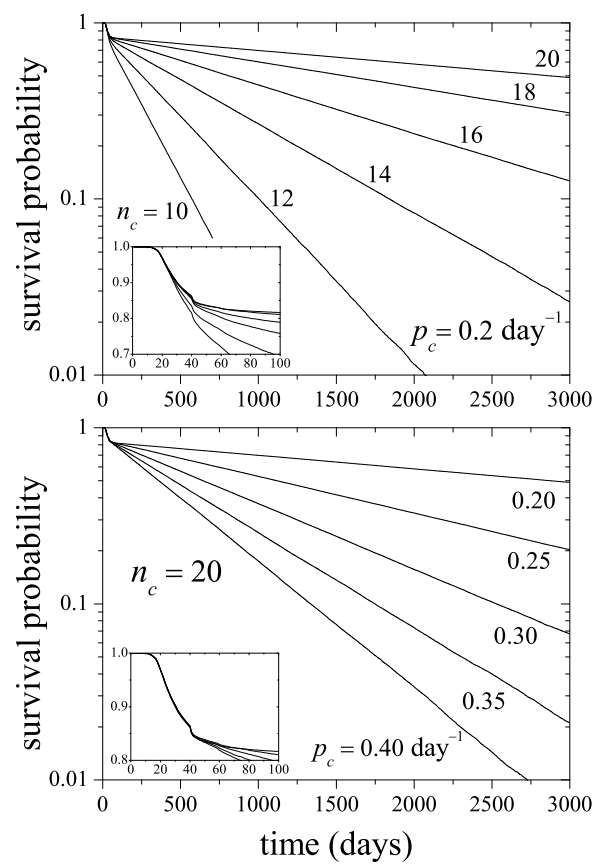

Fig. 3. Upper panel: The survival probability $P(t)$ as a function of time for $p_{c}=0.2$ day $^{-1}$ and several values of the awareness threshold $n_{c}$, calculated from series of $10^{5}$ realizations for each parameter set (log-linear scales). The inset shows a close-up for short times (linear-linear scales). Lower panel: As in the upper panel, for $n_{c}=20$ and several values of $p_{c}$.

The upper panel of Fig. 3 shows the survival probability $P(t)$ as a function of 
time in $\log$-linear scales, for $p_{c}=0.2$ day $^{-1}$ and several values of the awareness threshold $n_{c}$. The inset is a close-up for short times. Like for the average total population as a function of time (Fig. 2), we find that the survival probability exhibits two well defined regimes. For short times $(t \lesssim 30$ days, or a month), $P(t)$ is independent of the threshold $n_{c}$. In this regime, the survival probability is controlled by the spontaneous extinction of the first generation of lice, having never attained a sufficiently large number of mobile individuals. At the end of this period, some $10 \%$ of the colonies have become extinct. For long times $(t>100$ days, or about three months), on the other hand, an exponential tail develops. As expected, the slope is less steep as $n_{c}$ increases and longer durations become more likely. In the intermediate zone $\left(30 \lesssim t \lesssim 100\right.$ ), where the curves for different $n_{c}$ begin to diverge from each other, the wavy profile of $P(t)$ reveals the effect of the oscillations referred to in connection with the results shown in the lower panel of Fig. 1, which make extinction more probable when the initial generations conclude their life cycles.

Similar results are shown in the lower panel of Fig. 3, for a fixed awareness threshold $n_{c}=20$ and several values of $p_{c}$. Now, the short time regime where the survival probability is independent of $p_{c}$ extends up to $t \approx 45$ days, where slightly above $15 \%$ of the colonies have become extinct. For long times, the exponential tails are steeper as $p_{c}$ increases.

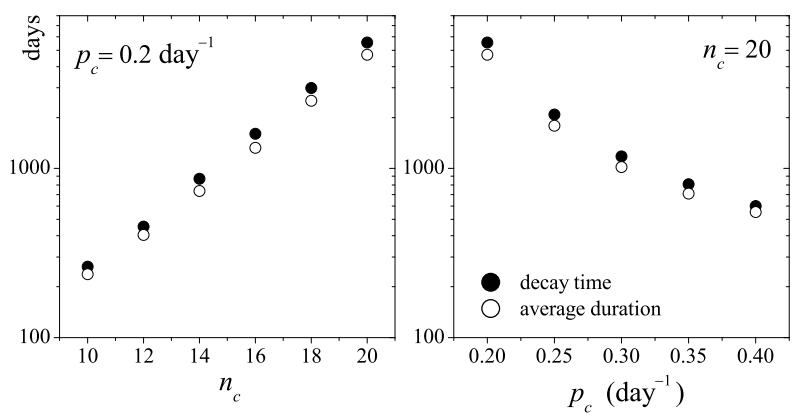

Fig. 4. Left panel: Decay time of the exponential tail of the survival probability ( $\tau$, full dots), and average duration of colonies ( $T$, empty dots) as functions of $n_{c}$ for $p_{c}=0.2$ day $^{-1}$, calculated from series of $10^{5}$ realizations for each parameter set. Right panel: As in the left panel, for $n_{c}=20$ and as functions of $p_{c}$.

The fact that $P(t)$ decays exponentially for long times implies that, similarly, the probability $p(u)$ that the duration of a colony equals $u$ is exponential for large $u: p(u) \sim \exp (-u / \tau)$. The characteristic decay time $\tau$ is the same for $p(u)$ as for $P(t)$. Results of the computation of $\tau$ from least-square fitting of the data displayed in Fig. 3 are shown in Fig. 4 as full dots. Moreover, we have evaluated the average colony duration $T$ using Eq. (2), obtaining the empty dots of Fig. 4. Note that, for each parameter set, the values of $\tau$ and $T$ are very similar to each other, with 
the former systematically above the latter. As a matter of fact, if $P(t)$ were a purely exponential function, $\tau$ and $T$ would exactly coincide. Due however to the deviations for short times (shown in the insets of Fig. 3), which imply a relatively high frequency of short durations, the average duration is shorter than the decay time. The difference is slightly larger for large $n_{c}$ and small $p_{c}$, where the decay times are longer.

\section{Lice populations on several hosts}

On the basis of the results obtained in the preceding section, we now move on to consider a lice population infesting a group of several hosts. As advanced in Section 2.2. contagion between hosts is described by the per-day probability $p_{s}$ that a louse "jumps" from one host to another. We disregard any underlying structure in the host group, so that contagion is equally probable between any pair of hosts. We recall that our main aim is to evaluate how the number of hosts $H$ affects the possibility that lice colonies survive by extending its spatial distribution.

Each plot in Fig. 5 represents the lice population as a function of time in a single host's head, out of group of $H=4$ hosts, with a jump probability $p_{s}=0.03$ day $^{-1}$. The awareness threshold is $n_{c}=12$ and the additional death probability due to grooming above the threshold is $p_{c}=0.2 \mathrm{day}^{-1}$. Initially, the infestation is confined to the host represented in the uppermost panel. At later stages, it is transmitted by contagion to the other three hosts. Note that, occasionally, the population in an individual host may fall to zero, and reappear later.

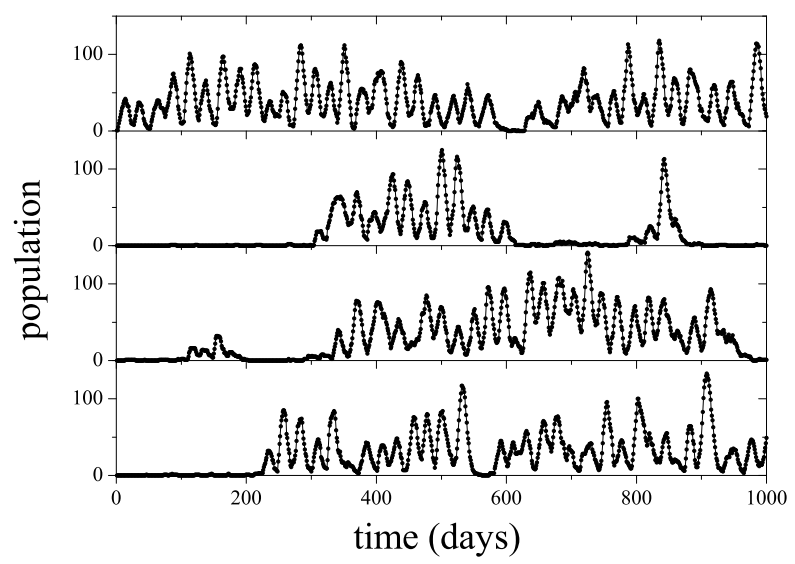

Fig. 5. Individual lice populations in a four-host group, with a jump probability between hosts $p_{s}=0.03$ day $^{-1}$. With this probability, each louse moves from its present host to any of the others. The awareness threshold is $n_{c}=12$, and the additional death probability due to grooming above the threshold is $p_{c}=0.2$ day $^{-1}$.

Rather straightforward arguments make it possible to predict that, under con- 
ditions where a single-host colony becomes extinct, mutual contagion between a sufficiently large group of hosts may however insure the survival of the lice population at arbitrary long times, and make the infestation endemic. Using for instance Levins time-continuous model for metapopulation dynamics [17, the number $h(t)$ of infested hosts, out of a group of $H$ hosts, is expected to vary as

$$
\dot{h}=-\frac{h}{\tau}+p_{h} h(H-h) .
$$

Here, $\tau$ is the average duration of a single-host colony (under the assumption that the colony's survival probability decays exponentially with time; see Section 31), and $p_{h}$ is the host-to-host contagion probability per time unit. The contribution of contagion is proportional to the number of infested and non-infested hosts, implying logistic evolution for $h(t)$. According to this model, $h(t)$ either decays to $h_{\infty}=0$ or approaches a finite value $h_{\infty}=H-\left(\tau p_{h}\right)^{-1}$ at asymptotically long times, depending on whether $H$ is lower or larger than $H_{c}=\left(\tau p_{h}\right)^{-1}$, respectively. In other words, whereas for $H<H_{c}$ the whole lice metapopulation becomes extinct, for $H>H_{c}$ it persists indefinitely, infesting a finite number of hosts. Note the reciprocal relation between the critical number of hosts and the contagion probability $p_{h}$. Moreover, for $H$ both above and below $H_{c}$ and for sufficiently long times, the number of infested hosts approaches its asymptotic value $h_{\infty}$ as $\left|h(t)-h_{\infty}\right| \sim \exp (-s t)$, with $s=$ $\left|H p_{h}-\tau^{-1}\right|$. The linear dependence of the exponential slope $s$ with the parameters $H$ and $p_{h}$ is a signature of the transcritical nature of the transition between extinction and survival in Levins model 18 .

In connection with our description - where lice colonies are not treated as metapopulations, but as multiagent populations - the host-to-host contagion probability in Eq. (3) must be associated with the jump probability $p_{s}$. Meanwhile, as we have seen in Section 3. the average duration of a single-host colony is determined by the whole set of parameters that define the louse's life history and the host's grooming. Whereas we do not foresee Levins model to reproduce our results at a quantitatively reliable level, we do expect to find a transition between extinction and survival of the lice population as the number of hosts $H$ grows, including the intuitive inverse relation between the critical number of hosts and the jump probability.

Figure 6 displays the mean lice population per host as a function of time, on groups of 2 to 10 hosts, with jump probability $p_{s}=0.01$ day $^{-1}$. As we see from Figs. 2 (upper panel) and 4 (left panel), the parameters $n_{c}=12$ and $p_{c}=0.2$ day $^{-1}$ correspond to a situation where a single-host colony becomes extinct within an average time of a few hundred days. For larger host groups, in contrast, Fig. 6 shows that the long-time population decay becomes slower as the number of hosts increases. For $H \gtrsim 8$, moreover, no decay can be perceived within the time range of the plot. The population seems to asymptotically reach a stationary level, which grows as the host group increases in size. In qualitative terms, this is precisely the behavior predicted by Levins model. 


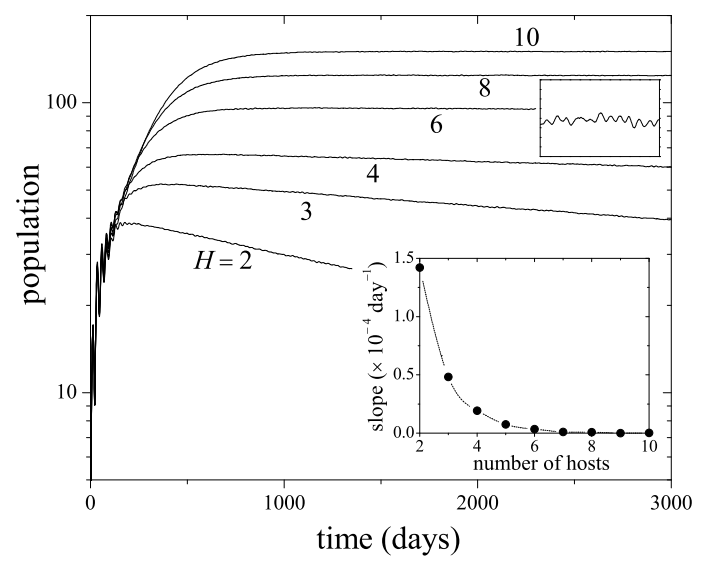

Fig. 6. Mean per-host population as a function of time, averaged over $10^{5}$ realizations, for $n_{c}=12$, $p_{c}=0.2$ day $^{-1}$, and jump probability $p_{s}=0.01$ day $^{-1}$, on groups of $H=2$ to 10 hosts. The upper inset is a close-up of the curve for $H=6$ for times between 2500 and 2800, amplifying by factors of 2 and 15 in the horizontal and vertical axes, respectively. The lower inset shows the asymptotic exponential slope $s$, obtained from least-square fittings for long times. The dotted curve is a spline interpolation for guiding the eye.

Quantitative differences are apparent, however, when we measure the exponential slope of the population decay as a function of the number of hosts. Results are displayed in the lower inset of Fig. 6. The slope decreases and approaches zero with a very smooth dependence on the number of hosts, suggesting a higher-order transition from extinction to survival. This smoothness jeopardizes the precision with which the transition point could be determined. Moreover, as discussed in more detail below, the slopes themselves are calculated up to a precision defined by the small irregular fluctuations of the population averaged over realizations, visible in the close-up of Fig. 6. The size of these fluctuations is controlled by the number of realizations ( $10^{5}$ in our case), and does not decrease as the number of hosts increases. This fact amounts to larger relative errors in the determination of smaller slopes, as $H$ grows. Finally, we cannot count upon improving our description of the transition by such standard methods as finite-size analysis as, being a system driven by the fluctuations of the population, the dynamics is not scalable to other population sizes or host numbers [19].

In view of these drawbacks, we analyze the transition from extinction to survival by an alternate method that, rather than focusing on the determination of critical points and exponents, seeks to characterize how the system approaches the transition as the control parameters are varied. Still, the method is based on the analysis of the average time dependence of the lice population, as shown by the curves of Fig. 6. For each of these curves, we define a normalized long-time slope as $S=\left(n_{\max }-n_{\min }\right) / n_{\max }$, where $n_{\max }$ is the maximum of the mean per-host population averaged over $10^{5}$ realizations, and $n_{\min }$ is the minimum reached by the 
same quantity in the time interval between the maximum and $t=3000$.

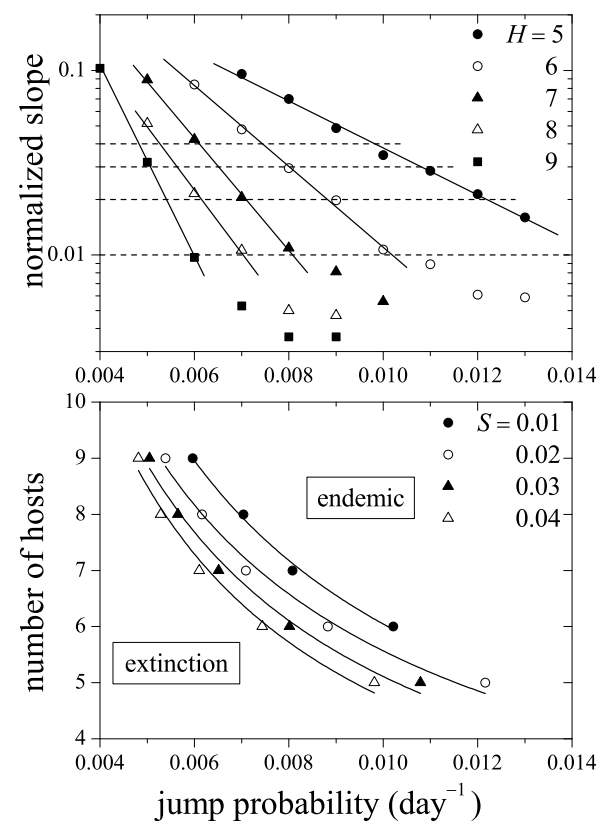

Fig. 7. Upper panel: Symbols stand for the normalized slope $S$ as a function of the jump probability $p_{s}$ for several values of the number of hosts $H$, calculated for the mean per-host lice population as a function of time, averaged over $10^{5}$ realizations. The awareness threshold is $n_{c}=12$ and the death probability above the threshold is $p_{c}=0.2$ day $^{-1}$. Full straight lines are exponential least-square fittings above $S=0.01$, for each value of $H$. Horizontal dashed lines are a set of selected levels of $S$, used to calculate the data shown in the lower panel. Lower panel: Symbols stand for the intersections between the selected levels of $S$ and the exponential fittings for each value of $H$, plotted in the $\left(p_{s}, H\right)$-plane. Curves are algebraic fittings of the form $H \propto p_{s}^{-\gamma}$. The exponent $\gamma$ ranges in the interval $(0.7,0.9)$ for the four curves.

Symbols in the upper panel of Fig. 7 stand for our determination of $S$ for host groups of size $H=5$ to 9 and several values of the jump probability $p_{s}$. As in Fig. [6] we have taken $n_{c}=12$ and $p_{c}=0.2$ day $^{-1}$. These results suggest that, for $S \gtrsim 0.01$, the normalized slope decreases exponentially with $p_{s}$, with steeper decays for larger values of $H$. Full straight lines in the plot correspond to leastsquare exponential fittings for each value of $H$, including all the data with $S \geq$ 0.01. The $R$-coefficients of the fittings are in all cases larger than 0.99. Below $S=$ 0.01 , clearly, the exponential dependence breaks down, and the normalized slope seems to approach a constant value as $p_{s}$ grows further. It is not difficult to realize that this regime results from the small fluctuations in the population as a function of time, caused by averaging over realizations, which are the dominant effect in the determination of $S$ when the curves become almost horizontal. Plausibly, the 
threshold could be lowered by averaging over more realizations, but the same effect would ultimately control the resulting value of $S$ for sufficiently small slopes. Our analysis, henceforth, focuses on the data above the threshold.

Once the exponential fittings of $S$ as a function of $p_{s}$ have been determined for each value of $H$, we choose a decreasing sequence of levels for the normalized slope, $S=0.04,0.03,0.02$, and 0.01 , indicated by the horizontal dashed lines in the upper panel of Fig. 7 For each of these levels, we analytically determine its intersection with each exponential fitting, thus defining a pair $\left(p_{s}, H\right)$. Each pair gives an estimation for the jump probability and the number of hosts that, on the average, are expected to give a long-time dependence of the lice population which decays with the corresponding slope $S$. The pairs $\left(p_{s}, H\right)$ determined by this procedure are plotted in the lower panel of Fig. 7.

Inspired by Levins model, which predicts for the transition point an interdependence of the form $H \propto p_{h}^{-1}$ between the number of hosts and the host-tohost contagion probability, we fit to the pairs $\left(p_{s}, H\right)$ an algebraic function of the form $H \propto p_{s}^{-\gamma}$ for each value of $S$. The resulting exponents are all in the range $\gamma=0.8 \pm 0.1$, with a slight tendency to decrease for small values of $S$. The $R-$ coefficients of the fittings are above 0.98 . While, from this analysis, we are not able to define a critical transition line in the parameter plane $\left(p_{s}, H\right)$, we can safely associate the region above the curves with the endemic regime of pediculosis, while the lower region corresponds to the extinction of the lice population.

Finally, we recall that the above numerical results for lice populations on several hosts hold for the choice $n_{c}=12$ and $p_{c}=0.2$ day $^{-1}$. A simple dimensionality argument makes it possible to conjecture how the results can be extended to different values of the parameters that characterize grooming above the threshold. In fact, the interdependence between the (non-dimensional) number of hosts $H$ and the jump probability $p_{s}$ (whose units are day ${ }^{-1}$ ) must involve an additional quantity with units of time, in order to cancel the units of $p_{s}$. In the long-time stage of the evolution, this quantity is the decay time $\tau$ of the one-host survival probability, introduced in Section 3. This decay time fixes the scale of the demographic processes whose competition with host-to-host contagion determines the transition between extinction and the endemic state. In turn, $\tau$ is determined by $n_{c}, p_{c}$, and the lifecycle parameters of lice. Note that the result obtained from Levins metapopulation equation for the critical number of hosts, $H_{c}=\left(\tau p_{h}\right)^{-1}$, agrees with this argument. In our model, it amounts to conjecture that the algebraic relation assumed to hold between $H$ and $p_{s}$ for constant $S$, and illustrated in the lower panel of Fig. (7) should be written as $H \propto\left(\tau p_{s}\right)^{-\gamma}$. The rather strong dependence of $\tau$ on both $n_{c}$ and $n_{p}$ (see Fig. (4) suggests in turn that small variations in any of these parameters may have important effects in the transition point. For instance, for $p_{c}=0.2 \mathrm{day}^{-1}$, changing the awareness threshold from $n_{c}=12$ to 14 duplicates the value of $\tau$. This implies that, for a given number of hosts, the same long-time dynamics is obtained with half the jump probability. 


\section{Conclusion}

Colonies of Pediculus humanus capitis, the head louse, typically consist of a relatively small number of individuals on each human host. This is due to the limiting intervention of the hosts themselves who, usually, do not tolerate the symptoms of pediculosis without actively contributing to lice mortality through grooming or other treatments. On a single host, this maintains the colony on the verge of extinction, likely to disappear by mere stochastic fluctuations in its population. Moreover, lice die within a few hours if they are removed from their hosts and cannot have a timely meal of blood. The continued prevalence of pediculosis along mankind's history - inherited, in turn, from other primates - must thus be explained taking into account the possibility of contagion from host to host. The head louse thus constitutes a remarkable example of survival by expansion of the spatial distribution: migration between different domains - the hosts' heads - makes it possible that the population, otherwise jeopardized by aggressive external factors, grows and becomes established.

To quantitatively characterize this phenomenon, we have here used a computational model based on realistic empirical data from the louse's life cycle. The model is an adaptation of a recently proposed mathematical formulation used to evaluate the effect of various treatment strategies for pediculosis [6. We have shown that, on the average, a lice colony infesting a single host never exceeds some tens of individuals, and takes a time of the order of several months to disappear by population fluctuations. When contagion between hosts becomes possible, on the other hand, a group of less than ten hosts in mutual contact already makes it possible that the lice population survives indefinitely. These quantitative results, however, have required to assign values to certain parameters for which there are no systematic empirical observations - namely, the host's awareness threshold, the effects of grooming on lice mortality, and the contagion probability. Although our choices sound to be reasonably realistic, at least in their order of magnitude, more precise data would help to sharpen the present conclusions.

It is interesting to remark that, despite of the complex fluctuation-controlled demographic dynamics of each individual colony, the average behavior of a lice population distributed over several hosts is well described - at least, qualitatively - by a simple metapopulation model which only takes into account the mean life of a colony on a single host and the contagion probability between hosts. This model predicts a reciprocal relation between the critical number of hosts necessary for the infestation to reach an endemic state and the contagion probability. In our more detailed description, the possibility of contagion is implicitly (but not directly) determined by the probability that each individual louse passes from one host to another. In spite of this difference, we also find an inverse relation between the number of hosts necessary to yield a given overall decay in the lice population and this "jump" probability, although the relation involves a different exponent. This suggests that, as in other models for distributed demography [5], the transition 
between extinction and the endemic state is ultimately controlled by a straightforward competition between the time scales of local population decay, on one side, and migration, on the other.

In our analysis, we have not considered any mechanism able to remove eggs from the hosts' hair. In this sense, we have been describing host groups with no access to pharmacological treatments whose use against pediculosis is standard in the present day - or groups that purposely avoid their use. The extension of the model to include such mechanisms is in principle self-evident, though it would require a quantitative evaluation of their efficacy. Taking into account that, on the average, some two thirds of the lice population are normally in the egg stage, effective removal of eggs should translate into a substantial decrease of the mean lifetime of a colony [6]. According to our results, this decrease makes a much larger host group necessary for the infestation to become endemic. The study of groups formed by many hosts, in turn, may require taking into account the structure and dynamics of social interactions inside each group, and their interplay with the epidemiology of pediculosis, an interesting topic toward which our work calls for being extended.

\section{Acknowledgments}

We thank Drs. Fabiana Laguna and Sebastián Risau Gusmán for their critical reading of the manuscript. Financial support from ANPCyT (PICT 2011-545) and SECTyP UNCuyo (Project 06/C403), Argentina, is acknowledged.

\section{References}

[1] J. D. Murray, Mathematical Biology I. An Introduction (Springer, Berlin, 2002).

[2] J. D. Murray, Mathematical Biology II. Spatial Models and Biomedical Applications (Springer, Berlin, 2003).

[3] R. Lande, S. Engen, and B.-E. Saether, Stochastic Population Dynamics in Ecology and Conservation (Oxford University Press, 2003).

[4] G. M. Mace, N. J. Collar, K. J. Gaston, C. Hilton-Taylor, H. R. Akçakaya, N. LeaderWilliams, E. J. Milner-Gulland, and S. N. Stuart, Quantification of extinction risk: IUCN's system for classifying threatened species, Conserv. Biol. 22 (2008) 1424-1442.

[5] M. Khasin, B. Meerson, E. Khain, and L. M. Sander, Minimizing the population extinction risk by migration, Phys. Rev. Lett 109, 138104 (2012).

[6] M. F. Laguna and S. Risau-Gusmán, Of lice and math: Using models to understand and control populations of head lice, PLoS ONE 6 (2011) e21848.

[7] N. Gratz, Human lice, their prevalence and resistance to insecticides (World Health Organization, Geneva, 1998).

[8] M. Takano-Lee, K. S. Yoon, J. D. Edman, B. A. Mullens, and J. M. Clark, In vivo and in vitro rearing of Pediculus humanus capitis (Anoplura: Pediculidae), J. Med. Entomol. 40 (2003) 628-635.

[9] R. Speare, D. V. Canyon, and W. Melrose, Quantification of blood intake of the head louse: Pediculus humanus capitis, Int. J. Dermat. 45 (2006) 543-546.

[10] M. A. Perotti, S. S. Catalá, A. Ormeño, M. Żelazowska, S. M. Biliński, and H. R. Braig, The sex ratio distortion in the human head louse is conserved over time, BMC Genetics 5 (2004) 10. 
[11] C. N. Burkhart and C. G. Burkhart, Fomite transmission in head lice, J. Amer. Acad. Dermat. 56 (2007) 1044-1047.

[12] D. V. Canyon and R. Speare, Indirect transmission of head lice via inanimate objects, Open Dermat. J. 4 (2010) 72-76.

[13] P. Stone, H. Wilkinson-Herbots, V. Isham, A stochastic model for head lice infections, J. Math. Biol. 56 (2008) 743-763.

[14] M. Takano-Lee, J. D. Edman, B. A. Mullens, and J. M. Clark, Transmission potential of the human head louse, Pediculus capitis (Anoplura: Pediculidae), Int. J. Dermat. 44 (2005) 811-816.

[15] D. V. Canyon, R. Speare, and R. Muller, Spatial and kinetic factors for the transfer of head lice (Pediculus capitis) between hairs, J. Invest. Dermat. 119 (2002) 629-631.

[16] S. Sonnberg, F. A. Oliveira, I. L. Araújo de Melo, M. M. de Melo Soares, H. Becher, J. Heukelbach, Ex vivo development of eggs from head lice (Pediculus humanus capitis), Open Dermat. J. 4 (2010) 82-89.

[17] I. Hanski, Single-species metapopulation dynamics, Bio. J. Linn. Soc. 42 (1991) 1738.

[18] P. G. Drazin, Nonlinear Systems (Cambridge University Press, Cambridge, 1994).

[19] K. Binder and D. W. Heermann, Montecarlo Simulation in Statistical Physics (Springer, Berlin, 1988). 